\title{
Methods of Administering Superimposed High-Frequency Jet Ventilation and the Associated Risk for Aspiration in a Model of Tracheal Bleeding
}

\author{
Andreas Nowak $^{\mathrm{a}}$ Taras Usichenko ${ }^{\mathrm{c}}$ Michael Wendt ${ }^{\mathrm{c}} \quad$ Eckart Klemm $^{\mathrm{b}}$ \\ Departments of anesthesiology and Intensive Care Medicine, Emergency Medicine and Pain Management, and \\ b Otorhinolaryngology, Head and Neck Surgery, and Plastic Surgery, Dresden Friedrichstadt Hospital, \\ Academic Teaching Hospital, Technical University of Dresden, Dresden, and ' $\mathrm{Department}$ of Anesthesiology and \\ Intensive Care Medicine, University Medicine of Greifswald, Greifswald, Germany
}

\section{Key Words}

Aspiration - Bleeding · Jet ventilation - Tracheal surgery

\begin{abstract}
Background: To determine the suitability of different superimposed high-frequency jet ventilation (SHFJV) application methods during tracheal bleeding. Objective: To determine the effect of SHFJV on the aspiration of blood during tracheal bleeding. Methods: A test lung was ventilated using SHFJV via a rigid endoscope, a jet laryngoscope and a 4-lumen jet catheter. Packed red blood cells (PRBCs) were injected into the artificial trachea caudally to the rigid endoscope and jet laryngoscope ventilation, and both caudally and cranially during ventilation via the 4-lumen jet catheter, and the migration of PRBCs during ventilation was studied using continuous video recording. Results: Migration of blood into the lower respiratory tract did not occur during SHFJV via the rigid endoscope and jet laryngoscope and via the 4-lumen jet catheter with the bleeding caudal to ventilation source. If the bleeding was cranial to the 4-lumen jet catheter ventilation, migration of blood into the lower respiratory tract was seen when reflux of blood reached the entrainment area.
\end{abstract}

From this area, blood is transported within the jet stream into the lower respiratory tract. Conclusions: SHFJV protects the lower respiratory tract from blood aspiration in case of tracheal bleeding. During SHFJV via the 4-lumen jet catheter, aspiration of blood only occurs if bleeding is localized cranial to the 4-lumen jet catheter ventilation. In case of heavy tracheal bleeding, the jet sources should be positioned cranial to the site of bleeding.

Copyright @ 2012 S. Karger AG, Basel

\section{Introduction}

Superimposed high-frequency jet ventilation (SHFJV) which does not require an endotracheal tube was developed specifically for use in laryngotracheal surgery. The application of SHFJV extends opportunities for surgeons, interventional pulmonologists and anesthesiologists to control the surgical field. SHFJV uses two simultaneous jet streams with different frequencies and is applied in the trachea using several tools, including jet laryngoscopes, rigid endoscopes, jet catheters and TwinStream jet ventilators (Carl Reiner GmbH, Vienna, Austria). SHFJV con-

\section{KARGER}

Fax +41613061234

E-Mail karger@karger.ch

www.karger.com
(C) 2012 S. Karger AG, Basel

$0025-7931 / 13 / 0851-0059 \$ 38.00 / 0$

Accessible online at:

www.karger.com/res
Andreas Nowak, MD, Department of Anesthesiology and Intensive Care Medicine Emergency Medicine and Pain Management, Dresden Friedrichstadt Hospital Academic Teaching Hospital, Technical University of Dresden

Friedrichstrasse 41, DE-01067 Dresden (Germany)

E-Mail nowak-an@khdf.de 

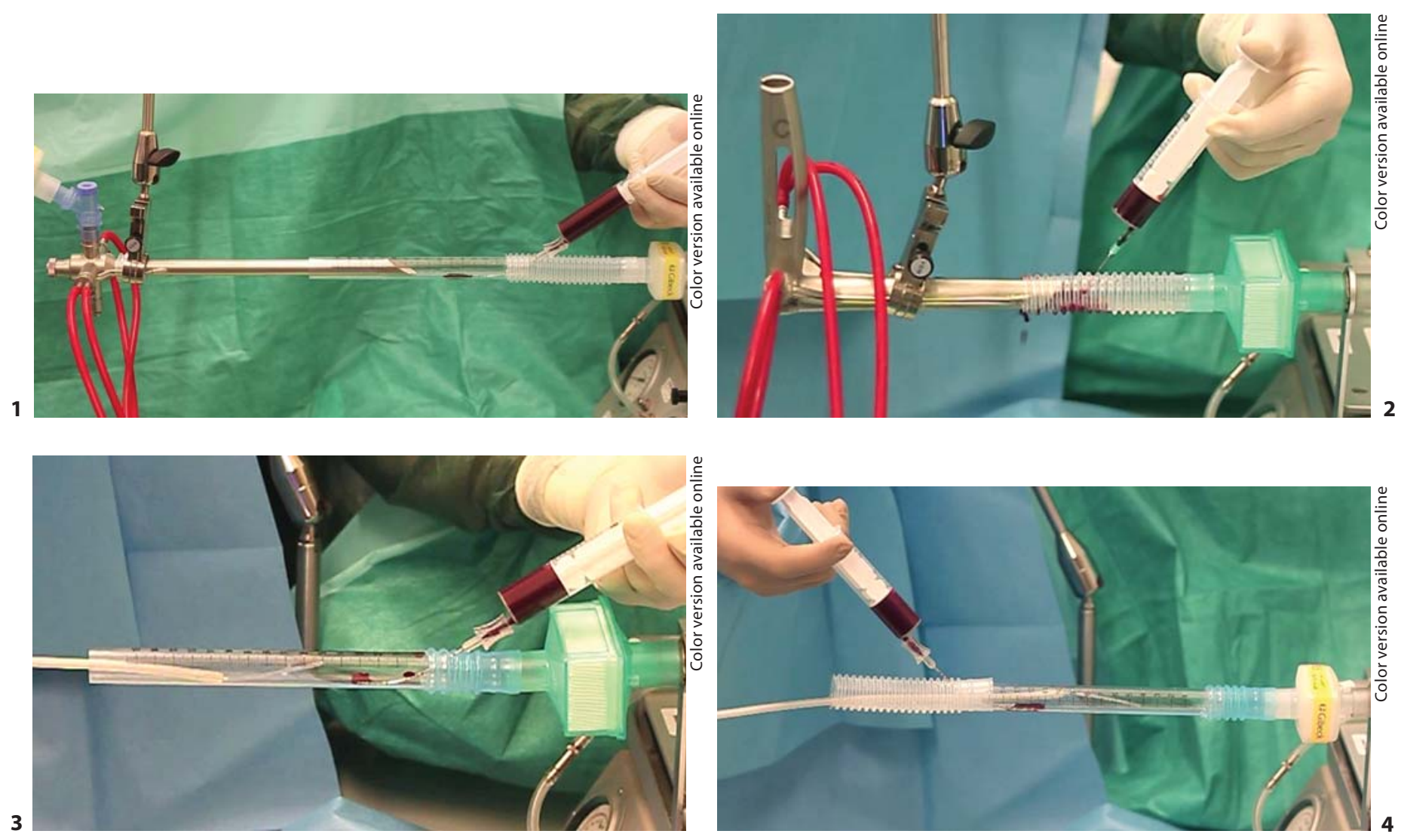

Fig. 1-4. Rigid endoscope (1), jet laryngoscope (2) and 4-lumen jet catheter $(\mathbf{3}, \mathbf{4})$ connected with an artificial trachea with caudal (1-3) and cranial (4) application of blood.

sists of a combination of pressure-limited low- (LF) and high-frequency (HF) jet ventilation with the application of sufficient tidal volumes and continuous oxygenation as well as ventilation during laryngotracheal interventions. SHFJV was developed in 1990 and has been used in clinical trials [1-5]. An important effect that occurs during transtracheal HF jet ventilation is the prevention of aspiration [6]. For example, hemoptysis is a frequently encountered symptom in the pulmonologist practice, with a spectrum from blood-streaked sputum to major bleeding with ensuing respiratory compromise. Major tracheal bleeding, although accounting for a minority of cases, should always be considered as a life-threatening condition that requires effective management of ventilation [7-9]. The purpose of this study was to explore the efficacy of SHFJV, which was administered tracheally via a rigid endoscope, jet tracheoscope or 4-lumen jet catheter, in preventing the aspiration of blood in the trachea in an experimental in vitro setting.

\section{Materials and Methods}

An adult training test lung (Michigan Instruments Inc., Grand Rapids, Mich., USA) with an attached graduated artificial trachea model (diameter $20 \mathrm{~mm}$ ) was used in all experimental tests. To protect the test lung from blood, we used heat and moisture exchangers (HME) between the artificial trachea and the test lung.

In trial 1, the test lung was ventilated via a rigid size-10 endoscope (Ref. No. CTNS-414-TBS; Carl Reiner GmbH) using SHFJV (TwinStream, Carl Reiner $\mathrm{GmbH}$ ) simultaneously via two integrated jet nozzles $[\mathrm{HF}=600 / \mathrm{min}$, inspiratory:expiratory ratio $(\mathrm{I}: \mathrm{E})=1: 1 ; \mathrm{LF}=12 / \mathrm{min}, \mathrm{I}: \mathrm{E}=1: 1$; driving pressure 1.0 bar at both nozzles; compliance of the ventilated test lung: 0.04 liters $/ \mathrm{cm}$ $\mathrm{H}_{2} \mathrm{O}$ ]. The airway pressures [positive end expiratory pressure $(\mathrm{PEEP}) /$ peak inflation pressure (PIP)] of the test lung were measured $(\mathrm{PIP}=14.2$ mbar, $\mathrm{PEEP}=6.2$ mbar; fig. 1 ).

In trial 2, the test lung was ventilated via a size- $\mathrm{C}$ jet tracheoscope (Ref. No. CTNS-330-001; Carl Reiner GmbH) using SHFJV with the parameters as in trial 1 . The airway pressures (PIP/PEEP) of the test lung were measured (PIP $=10.0 \mathrm{mbar}, \mathrm{PEEP}=6.0 \mathrm{mbar}$; fig. 2). 


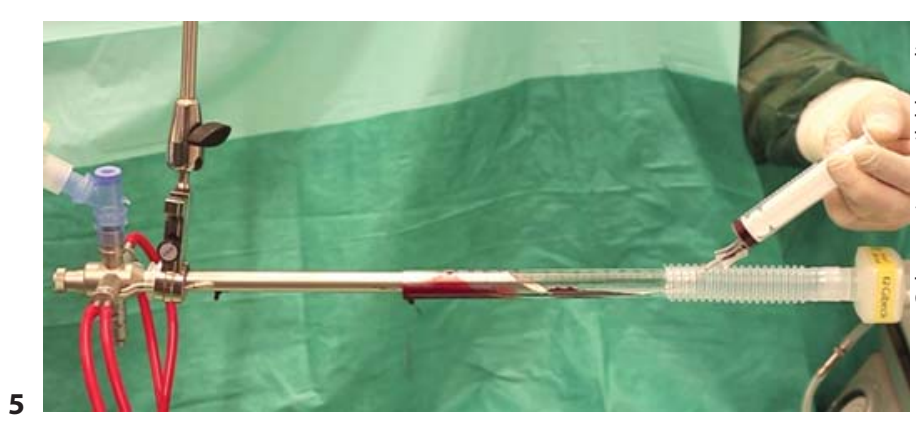

Fig. 5, 6. During the use of SHFJV, no backflow of blood from the trachea into the rigid endoscope (5) and jet laryngoscope (6) occurred. Transportation of blood into the lower respiratory tract did also not occur. Backflow of the exhaled gas and reflux of the

In trial 3, the test lung was ventilated via a 4-lumen jet catheter (Ref. No. CTFS-544-010; Carl Reiner GmbH) using SHFJV with the same frequency as in trial 1 , but modified $\mathrm{I}: \mathrm{E}=1: 1.5 ; \mathrm{LF}=12$ / min, I:E = 1:1.5; driving pressure LF nozzle: 2.5 bar and HF nozzle: 1.5 bar; compliance of the ventilated test lung: 0.04 liters $/ \mathrm{cm}$ $\mathrm{H}_{2} \mathrm{O}$ ). The airway pressures (PIP/PEEP) of the test lung were measured (PIP = 5.1 mbar, PEEP = 1.9 mbar; fig. 3 ).

In trial 4, $10 \mathrm{ml}$ of packed red blood cells (PRBCs) were injected cranial to a 4-lumen jet catheter during jet ventilation (over a period of $30 \mathrm{~s}$ ) in a graduated trachea model, and the migration of the PRBCs during breathing with SHFJV was documented by continuous video recording (fig. 4).

In trial 5, $10 \mathrm{ml}$ of PRBCs were introduced caudal to the (i) rigid endoscope; (ii) jet tracheoscope and (iii) 4-lumen jet catheter ( 5 tests for each source of jet ventilation) over a period of $30 \mathrm{~s}$ in a graduated trachea model, and the migration of the PRBCs during breathing with SHFJV was documented by continuous video recording (fig. 1-3).

\section{Results}

In our experimental simulation of a bleeding trachea, no backflow of blood occurred from the trachea into the endoscopes during the use of SHFJV with the rigid endoscope and the jet laryngoscope or the 4-lumen jet catheter with caudal application of blood. These results were reproducible in a 5-test series. Transportation of blood into the lower respiratory tract did not occur in cases with jet ventilation over the rigid endoscope or jet laryngoscope (fig. 5, 6). During the caudal introduction of blood and use of SHFJV with the 4-lumen jet catheter, blood swirling occurred within the trachea model (fig. 7). During the use of the 4-lumen jet catheters with caudal introduction of blood, the HME between the artificial trachea and the test lung remained untouched by blood until the reflux of

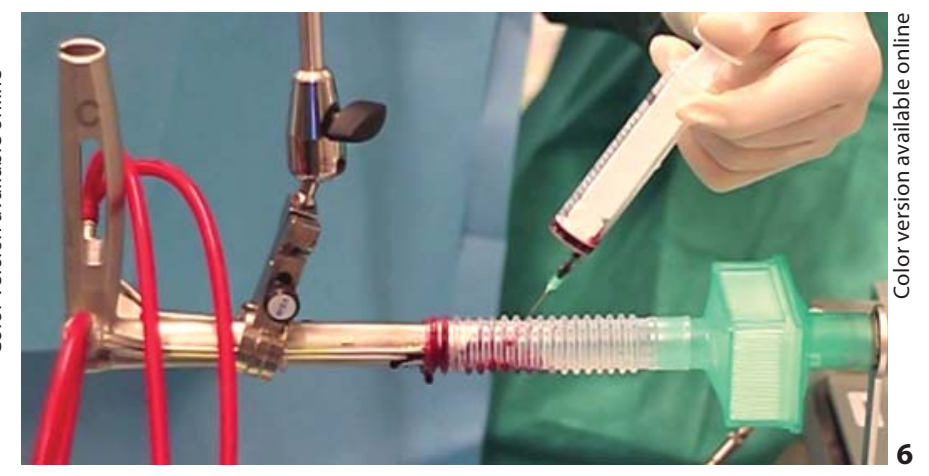

injected blood was caused by the remaining leakage between the rigid endoscope and the trachea. Additionally, continuous migration of injected blood towards the larynx was observed on the wall of the trachea.

blood reached the entrainment area. From this area, blood was transported within the jet stream into the lower respiratory tract. Additionally, continuous migration of injected blood toward the larynx was observed on the tracheal wall (fig. 7). During the application of blood and use of SHFJV with the 4-lumen jet catheter with cranial application of blood, swirling of blood occurred within the entire trachea model, and blood was also transported into the lower respiratory tract. The HME was rapidly filled with blood (fig. 8). Continuous migration of injected blood toward the larynx was simultaneously observed on the tracheal wall.

\section{Discussion}

Our results demonstrate that SHFJV prevents the aspiration of blood under the given conditions if the bleeding occurs caudal to the source of jet ventilation or if the bleeding is not localized within the stream of entrainment.

The main aim of the experimental simulation was to elucidate the flow process during SHFJV within the trachea as well as its interaction with bleeding during ventilation. This experimental simulation offers an outstanding way to understand fluid flow under highly transient conditions, such as during the application of SHFJV. However, the results of this experimental simulation strongly rely on the applied models and boundary conditions. Of course, the effects of SHFJV in case of heavy bleeding in deeper airway sections require further investigation. Because of Bernoulli's law, a pressure gradient generated through the openings of the airway dictates the 


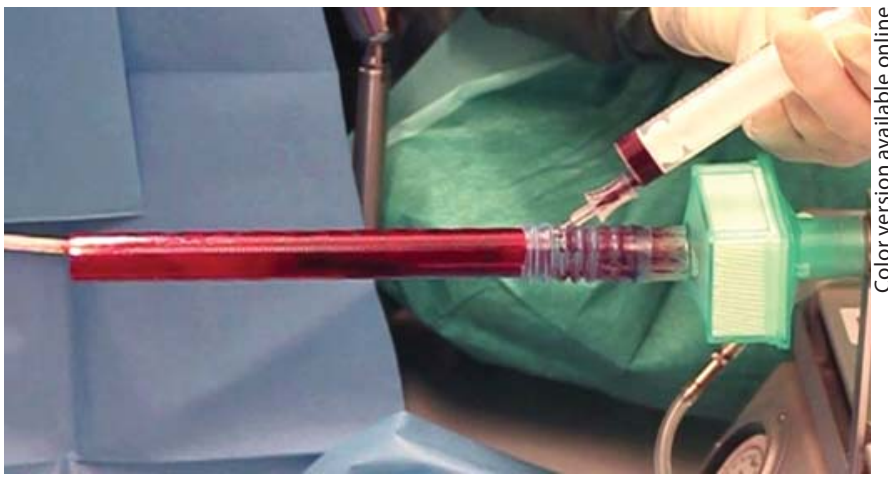

Fig. 7. During the application of blood and use of SHFJV with the 4-lumen jet catheter with caudal application of blood, swirling of blood within the trachea model occurred. The HME between the artificial trachea and the test lung remained untouched by blood until the reflux of blood and backflow of exhaled gas reached the entrainment area. From this area, blood was transported within the jet stream into the lower respiratory tract. Moreover, continuous migration of blood towards the larynx was observed on the tracheal wall.

direction of the fluid flow. For all forms of jet ventilation, gas leakage in conjunction with pressure difference between the inside of the airway and the outer region is responsible for the flow direction and speed of respiratory gas and blood (e.g. in the case of heavy tracheal bleeding).

Therefore, experimental investigations show that a backflow of air and blood from the trachea into the endoscopes in the case of tracheal bleeding during SHFJV is unlikely under the given boundary conditions. The mechanisms underlying the prevention of pulmonary aspiration likely involve the continuous gas outflow from the lungs. This forceful gas outflow serves as a pneumatic valve to prevent aspiration. When using the 4-lumen jet catheters, it is important to ensure that the jet catheter is positioned cranial to the source of bleeding within the trachea. If the jet catheters are positioned caudal to the source of tracheal bleeding, the blood will be transported into the lower respiratory tract. Our model enables a simple comparison of aspiration risk during peri-interventional tracheal bleeding using three methods of SHFJV application. This type of study would not be feasible if performed in patients at risk for inadequate oxygenation and ventilation. However, we appreciate that there may be differences between our model and the in vivo situation in terms of airway anatomy, controlled delivery of SHFJV in a shared airway during operational intervention and specific patient factors. Depending on the diameter of the instruments introduced (i.e. to perform tracheal hemo-

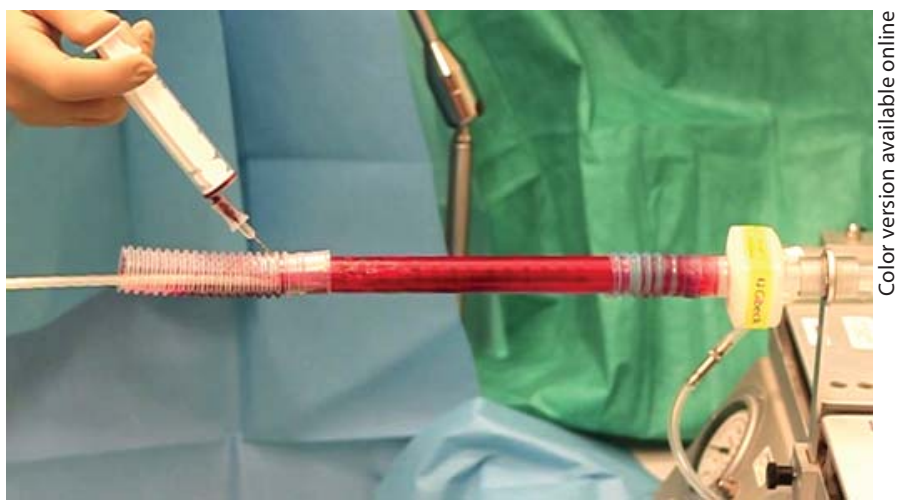

Fig. 8. During the use of SHFJV with 4-lumen jet catheters and cranial introduction of blood, transportation of blood into the lower respiratory tract was observed. Swirling of blood within the entire trachea model occurred. Continuous migration of blood towards the larynx was observed on the wall of the artificial trachea.

stasis, laser therapy) the entrainment as well as the gas flow in the trachea and, consequently, the effects described may be reduced. Adding an HME to a respiratory system may cause a small increase in airway resistance. HMEs may also reduce the pressure amplitude generated by the ventilator. However, these differences do not affect the high gas flow during HF ventilation modes like SHFJV. Regarding the flow characteristics and blood flow direction, the small increase in airway resistance with HMEs inserted can be ignored in this model. The horizontal experimental setup, the low elasticity of the artificial trachea and the temporary observation interval are noted as limitations of this model.

\section{Conclusion}

In this trachea model, SHFJV was associated with protection against the aspiration of blood if the tracheal bleeding was caudal to the source of jet ventilation or if the bleeding was not localized within the entrainment stream. The jet device has to be positioned cranial to the bleeding source. If the tracheal bleeding occurs cranial to the jet source, blood will be aspirated during SHFJV. This difference is attributable to the entrainment of air, which transports the blood into the lower respiratory tract. The implication of our results for the practicing anesthesiologist is that in the case of heavy tracheal bleeding, the jet 
sources should be positioned cranial to the bleeding site. The findings of this study also lead to the conclusion that a 4-lumen-jet catheter should not be used if relevant tracheal bleeding is anticipated. In summary, SHFJV may reduce the risk of aspirating blood and hence the potential for asphyxia in patients that undergo tracheal surgery and have a potential risk of heavy bleeding into the respiratory tract.

\section{Financial Disclosure and Conflicts of Interest}

The authors have no funding, financial relationships or conflicts of interest to disclose.

\section{References}

1 Aloy A, Schachner M, Cancura W: Tubeless translaryngeal superimposed jet ventilation. Eur Arch Otorhinolaryngol 1991;248:475478.

-2 Bacher A, Lang T, Weber J, Aloy A: Respiratory efficacy of subglottic low-frequency, subglottic combined-frequency, and supraglottic combined-frequency jet ventilation during microlaryngeal surgery. Anesth Analg 2000;91:1506-1512.
-3 Bacher A, Pichler K, Aloy A: Supraglottic combined frequency jet ventilation versus subglottic monofrequent jet ventilation in patients undergoing microlaryngeal surgery. Anesth Analg 2000;90:460-465.

4 Kraincuk P, Kormoczi G, Prokop M, Ihra G, Aloy A: Alveolar recruitment of atelectasis under combined high-frequency jet ventilation: a computed tomography study. Intensive Care Med 2003;29:1265-1272.

5 Rezaie-Majd A, Bigenzahn W, Denk DM, Burian M, Kornfehl J, Grasl MCh, Ihra G, Aloy A: Superimposed high-frequency jet ventilation (SHFJV) for endoscopic laryngotracheal surgery in more than 1500 patients. Br J Anaesth 2006;96:650-659.
6 Klain M, Keszler H, Stool S: Transtracheal high frequency jet ventilation prevents aspiration. Crit Care Med 1983;11:170-172.

7 Rose AS, Mathur PN: Endobronchial capillary hemangioma: case report and review of the literature. Respiration 2008;76:221-224.

8 Faguang I, Deguang M, Dongling C, Enquing F, Yonghong X, Tonggang L: Severe complications of bronchoscopy. Respiration 2008;76:429-433.

9 Sakr L, Dutau H: Massive hemoptysis: an update on the role of bronchoscopy in diagnosis and management. Respiration 2010;80:3858. 